\title{
The Comparative Analysis between Truss Aqueduct and Cable-stayed Aqueduct
}

\author{
Xue-Jian CHEN, Dong-Lin LIU, Li-Ting WANG, Yong YE*, Run ZHOU, \\ Zhen-Dong ZHANG
}

College of Hydraulic \& Environmental Engineering, Three Gorges University, Yichang, Hubei, China

yyeeong@aliyun.com

${ }^{*}$ Corresponding author

Keywords: Truss Aqueduct, Cable-stayed Aqueduct, Structure Calculation, Mechanical Analysis.

\begin{abstract}
Aqueduct is a kind of overhead conveyance structure crossing the river, road, thrust, valley and so on. Analyzing the truss aqueduct and the cable-stayed aqueduct through model design, structure calculation and comparative analysis. Through the static load experiment, bearing quality of cable-stayed aqueduct model is relatively large, showing that its bearing capacity is better than the truss aqueduct. Through the calculation of structural solver, get the internal force distribution and deformation rules of two kinds of models which are basically the same as experimental results. On the same loading condition, deformation of truss aqueduct is slightly larger than the cable-stayed aqueduct, providing references for the aqueduct's structure selection and engineering application.
\end{abstract}

\section{Model Design}

With characteristics of high rigidity and small deformation, the truss structure does not generate horizontal reaction under the action of load. So it's simple to complete the model calculation. The model of truss aqueduct is girder truss aqueduct. Span length is $1000 \mathrm{~mm}$, width $200 \mathrm{~mm}$, height $110 \mathrm{~mm}$. Slot body choose the best rectangular hydraulic section[1], because it's convenient to construction and the material requirements is low. In addition, the influence of aqueduct's stability by wind and horizontal loads can be reduced by increasing the bar. Rectangular groove body width is $200 \mathrm{~mm}$, deep $120 \mathrm{~mm}$. Meet the design requirements(the depth to width ratio $\mathrm{H} / \mathrm{B}=0.6-0.8$, high span ratio $\mathrm{h} / \mathrm{f}=0.1-0.2)$ (concrete size is shown in Fig.1.1, unit $\mathrm{mm}$ ).

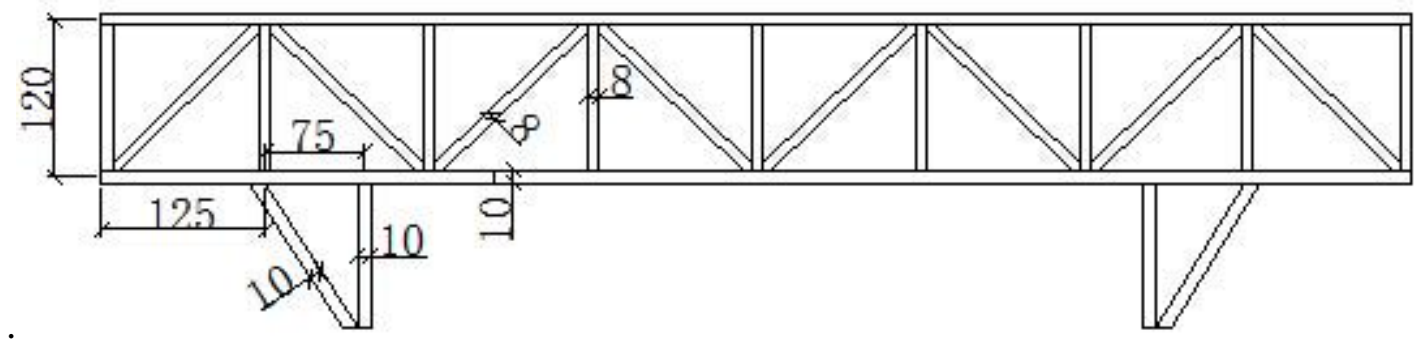

Fig.1.1 The Vertical View of Truss Model

At the same time, the girder of truss model is in the form of overhanging beam. When the simply supported beam is under the uniform load and distance between bearing and rod is $0.207 \mathrm{~L}$, through the calculation of structural solver, it is known that the maximum positive moment on the beam is equal to the maximum negative moment. At this time the maximum value of the beam's bending moment is minimal, so it can effectively improve the bearing capacity.

Because of cables' support, the cable-stayed aqueduct's [2] distribution of internal force in girder becomes reasonable and beam bending moment is greatly reduced. It increases the carrying capacity of model as well. In addition, the cable-stayed aqueduct is a kind of statically indeterminate structure and its stability is good. Cable-stayed aqueduct model: the span length $1000 \mathrm{~mm}$, width $200 \mathrm{~mm}$, height $110 \mathrm{~mm}$, the rectangular groove body width $200 \mathrm{~mm}$, depth $120 \mathrm{~mm}$. (The basic 
dimension of groove body is consistent to the truss aqueduct and they contrast.) Cable-stayed structure is layout by suspension system for radiation type[3]. (The specific size is shown in Fig. 1.2, unit $\mathrm{mm})$.

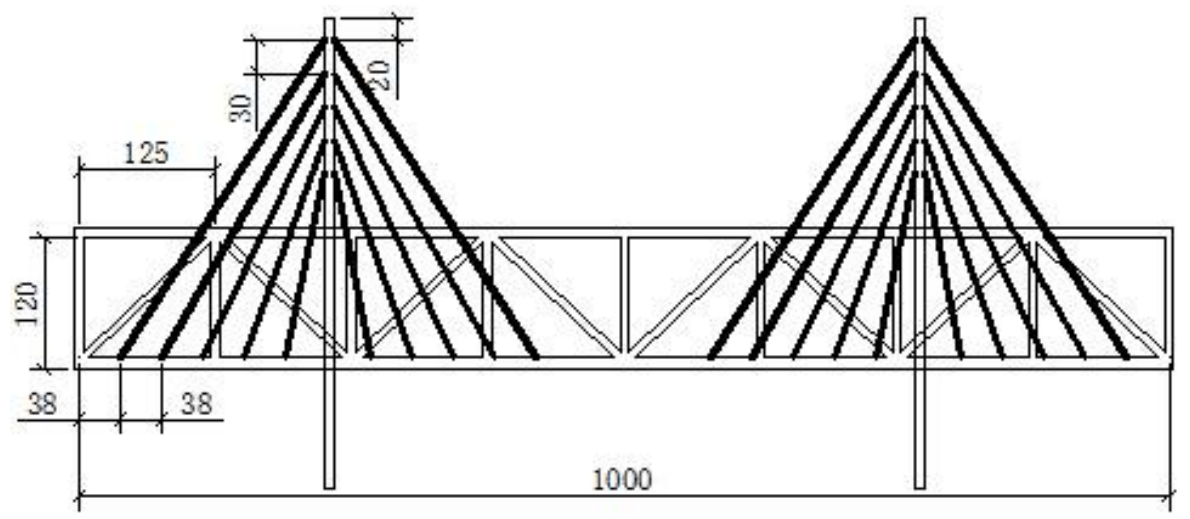

Fig.1.2 The Vertical View of Cable-stayed Model

\section{Model Experiment}

In order to carry out the water bearing test, the white cardboards are used to cover the interior of the aqueduct. The aqueduct's bottom and sides are sealed up to form a rectangular groove. Then spread preservative film on the white cardboard to prevent water from soaking white cardboard and rod so as to reduce the bearing capacity. Before the experiment, weigh the truss model and the cable-stayed model. Their respectively quality is $443.5 \mathrm{~g}$ and $482.2 \mathrm{~g}$. In the experiment, slowly add water while loading data. Each time when entry $1 \mathrm{~kg}$, measuring displacement of the control point in longitudinal beam. Through the experiment, the stringers' maximum displacement of two models occurred in the mid span position of beam. Displacement of span's midpoint under different load can be known.

Tab.2.1 The Load and Displacement of Water Tab.2.2 The Load and Displacement of the Iron Core

\begin{tabular}{|c|c|c|}
\hline \multirow{2}{*}{ Load $/ \mathrm{kg}$} & \multicolumn{2}{|c|}{ displacement $/ \mathrm{mm}$} \\
\cline { 2 - 3 } & Truss type & cable-stayed \\
\hline 10 & 2.5 & 1.7 \\
\hline 15 & 3.2 & 2.5 \\
\hline 20 & 4.4 & 3.0 \\
\hline 24 & 5.4 & 4.1 \\
\hline
\end{tabular}

\begin{tabular}{|c|c|c|}
\hline \multirow{2}{*}{ Load /kg } & \multicolumn{2}{|c|}{ displacement /mm } \\
\cline { 2 - 3 } & Truss type & cable-stayed \\
\hline 30 & 7.2 & 5.2 \\
\hline 40 & 9.1 & 6,8 \\
\hline 48 & 11.2 & 8.9 \\
\hline 57 & & 10.6 \\
\hline
\end{tabular}

When the water is full, the bearing capacities of the two models are $24 \mathrm{~kg}$. Although the two models has deformed, they are not destroyed, nor reaching the ultimate bearing capacity. In order to get the model of ultimate carrying capacity, bearing test is made with greater density of iron and obtained the displacement of the span's midpoint in the greater loads. The results are shown in the table 2.1-2. From them it's known that the ultimate bearing capacity of truss model and the cable-stayed type model are respectively $48 \mathrm{~kg}$ and $57 \mathrm{~kg}$.

\section{Mechanical Calculation}

\section{Truss Aqueduct Simplified and Stress Calculation}

The model material is ABS plastic tube. The elastic modulus is 2.2GPa, Poisson's ratio 0.394, density $1.06 \mathrm{~g} / \mathrm{m}^{3}$, tensile strength is $30 \mathrm{MPa}$, compressive strength $80 \mathrm{MPa}$. Size of main beam and support structure is $10 \times 10$ (unit: $\mathrm{mm}$ ), the rest of rod parts are made of size $8 \times 8$ (unit: $\mathrm{mm}$ ). The water pressure at the bottom of the aqueduct can be simplified to uniform load, bearing by two 
longitudinal beams. When the load of model is $\mathrm{mg}$, the uniform load subjected to longitudinal bear is $q=\frac{m g}{2 l}$. Because the aqueduct is symmetry about the longitudinal symmetry plane, it is reasonable to simplify a space structure as a plane structure. Therefore it's possible to establish the mechanical calculation model of aqueduct using structural solver and solve the displacement as well as internal force. Making use of the structural solver to establish truss aqueduct model, getting displacement of stringer cross point under different load, which is shown in table 3.1. Full load deformation diagram is shown in Fig. 3.1.

Tab.3.1 Displacement of the Midpoint of the Longitudinal Beam cross under Different Load

\begin{tabular}{|c|c|c|c|c|c|c|c|}
\hline load $/ \mathrm{kg}$ & 10 & 15 & 20 & 24 & 30 & 40 & 48 \\
\hline $\begin{array}{c}\text { displaceme } \\
\text { nt } / \mathrm{mm}\end{array}$ & 1.8940 & 2.8409 & 3.7879 & 4.5185 & 5.6819 & 7.5758 & 9.091 \\
\hline
\end{tabular}

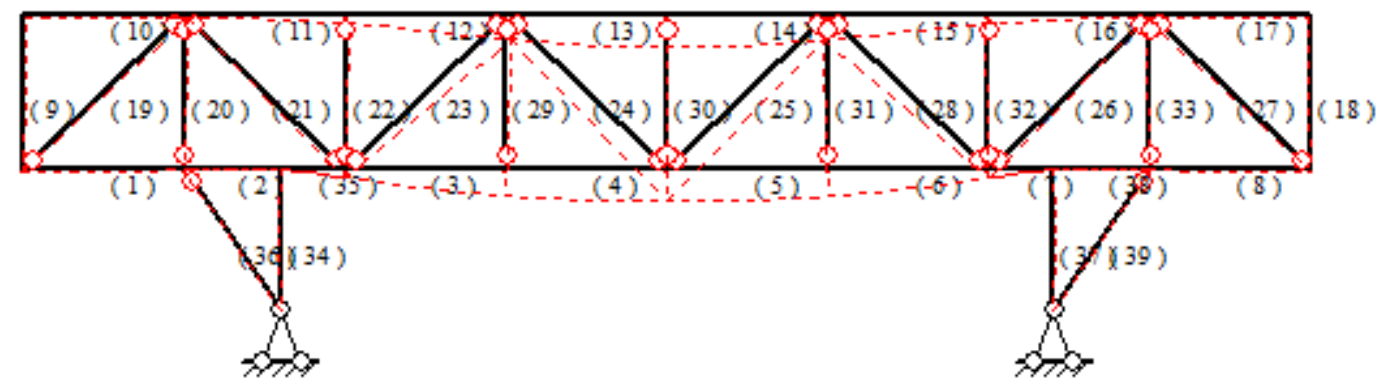

Fig.3.1 Deformation Map

In order to meet the bearing capacity of structure, it is necessary to check on the structure strength when it is loaded. According to the force calculation of structure solver, the checking point is unit 3, right endpoint of unit 13, unit 23, endpoint of unit 34 and left endpoint of unit 35.

For unit 3: $N=45.12 N, N=-60.22 N$ $\sigma_{t}=\frac{N}{A}+\frac{M}{W}=\frac{45.12}{100}+\frac{0.57 \times 1000 \times 6}{10 \times 10^{2}}=3.94 \mathrm{MPa}\langle 30 \mathrm{MPa}$, Meet the strength requirements.

For right endpoint of unit 13: $N=-52.54 N, M=0.27 N \cdot m$ $\sigma_{t}=\frac{N}{A}+\frac{M}{W}=\frac{-52.54}{64}+\frac{0.27 \times 1000 \times 6}{8 \times 8^{2}}=2.3 M P a\langle 30 M P a$, $\sigma_{c}=\frac{N}{A}+\frac{M}{W}=\frac{52.54}{64}+\frac{0.27 \times 1000 \times 6}{8 \times 8^{2}}=4.0 M P a\langle 80 M P a$, Meet the strength requirements.

For unit 23: $N=-60.22 N$ $\sigma_{c}=\frac{N}{A}=\frac{60.22}{64}=1.0 \mathrm{MPa}\langle 80 \mathrm{MPa}$, Meet the strength requirements.

For endpoint of unit 34: $N=-93.04 N, M=1.08 \mathrm{~N} \cdot \mathrm{m}$ $\sigma_{t}=\frac{N}{A}+\frac{M}{W}=\frac{-93.04}{100}+\frac{1.08 \times 1000 \times 6}{10 \times 10^{2}}=6.4 M P a\langle 30 M P a$, $\sigma_{c}=\frac{N}{A}+\frac{M}{W}=\frac{93.04}{100}+\frac{1.08 \times 1000 \times 6}{10 \times 10^{2}}=7.4 \mathrm{MPa}\langle 80 \mathrm{MPa}$, Meet the strength requirements.

For left endpoint of unit 35: $N=-6.81 N, M=2.75 N \cdot m$ $\sigma_{t}=\frac{N}{A}+\frac{M}{W}=\frac{-6.81}{100}+\frac{2.75 \times 1000 \times 6}{10 \times 10^{2}}=16.4 M P a\langle 30 M P a$, $\sigma_{c}=\frac{N}{A}+\frac{M}{W}=\frac{6.81}{100}+\frac{2.75 \times 1000 \times 6}{10 \times 10^{2}}=16.6 \mathrm{MPa}\langle 80 \mathrm{MPa}$, Meet the strength requirements. 


\section{Cable-stayed Aqueduct Simplified and Stress Calculation}

The cable of model is made of nylon rope. The elastic modulus is $3.2 \mathrm{GPa}$, diameter $0.72 \mathrm{~mm}$, tensile strength $70 \mathrm{MPa}$. The longitudinal beams and the support structure are made with $10 \times 10$ (unit: $\mathrm{mm}$ ) ABS plastic pipes. Use structural solver to establish cable-stayed aqueduct model. Get displacement of stringer cross point under different load, which is shown in table 3.2. Full load deformation diagram is shown in Fig. 3.2.

Tab.3.2 Displacement of the Midpoint of the Longitudinal Beam cross under Different Load

\begin{tabular}{|c|c|c|c|c|c|c|c|c|}
\hline load $/ \mathrm{kg}$ & 10 & 15 & 20 & 24 & 30 & 40 & 48 & 57 \\
\hline $\begin{array}{c}\text { displacem } \\
\text { ent } / \mathrm{mm}\end{array}$ & 1.4184 & 2.1276 & 2.8368 & 3.4041 & 4.2552 & 5.6735 & 6.8082 & 8.80848 \\
\hline
\end{tabular}

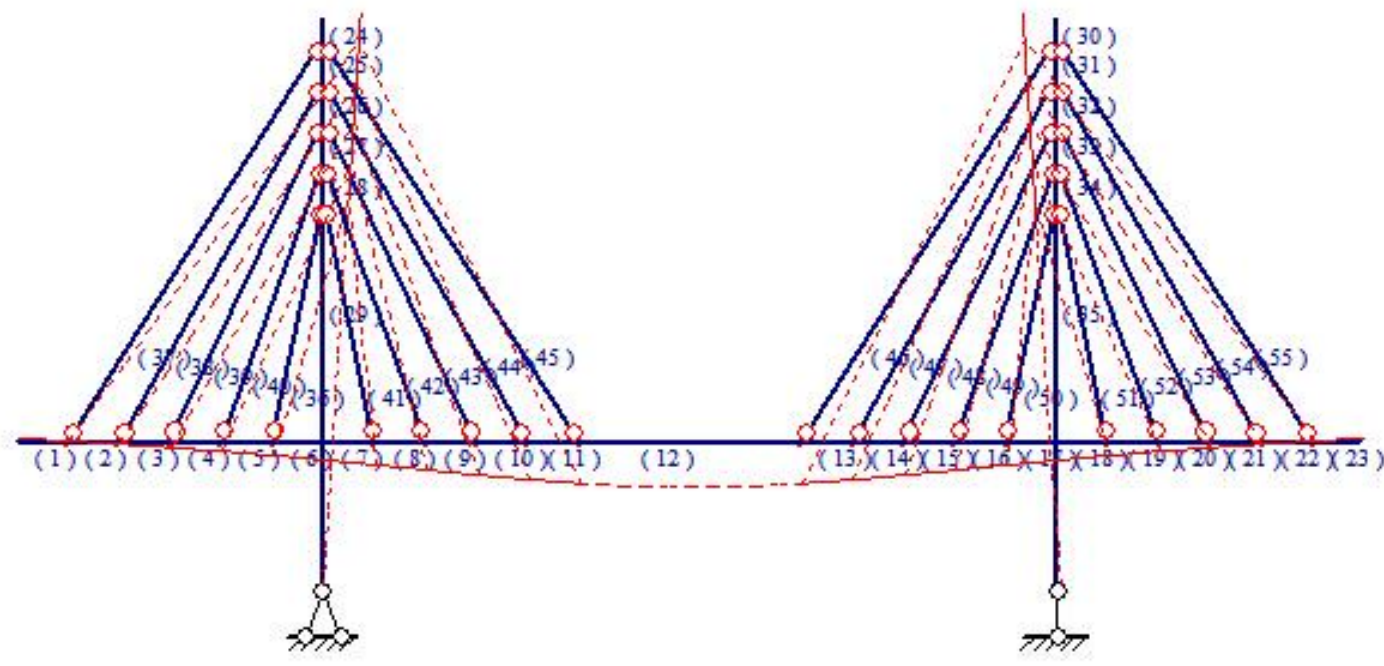

Fig.3.2 Deformation Map

According to the force calculation of structure solver, the checking point left endpoint of unit 5, left endpoint of unit 6, midpoint of unit 12, unit 29 and unit 36.

For left endpoint of unit 5: $N=-23.72 N, M=-0.9 N \cdot m$ $\sigma_{t}=\frac{N}{A}+\frac{M}{W}=\frac{-23.72}{100}+\frac{0.9 \times 1000 \times 6}{10 \times 10^{2}}=5.2 \mathrm{MPa}\langle 30 \mathrm{MPa}$, $\sigma_{c}=\frac{N}{A}+\frac{M}{W}=\frac{23.72}{100}+\frac{0.9 \times 1000 \times 6}{10 \times 10^{2}}=5.2 \mathrm{MPa}\langle 80 \mathrm{MPa}$, Meet the strength requirements.

For left endpoint of unit $6: \quad N=-28.18 N, M=-0.86 N \cdot m$ $\sigma_{t}=\frac{N}{A}+\frac{M}{W}=\frac{-28.18}{100}+\frac{0.86 \times 1000 \times 6}{10 \times 10^{2}}=3.9 M P a\langle 30 M P a$, $\sigma_{c}=\frac{N}{A}+\frac{M}{W}=\frac{28.18}{100}+\frac{0.86 \times 1000 \times 6}{10 \times 10^{2}}=5.4 \mathrm{MPa}\langle 80 \mathrm{MPa}$, Meet the strength requirements.

For midpoint of unit 12: $\quad \mathrm{N}=0, \quad M=3.7 \mathrm{~N} \cdot \mathrm{m}$ $\sigma_{t}=\frac{M}{W}=\frac{3.7 \times 1000 \times 6}{10 \times 10^{2}}=22.2 M P a\langle 30 M P a$, Meet the strength requirements.

For unit 29: $N=-139.65 N$ $\sigma_{c}=\frac{N}{A}=\frac{139.65}{100}=1.4 \mathrm{MPa}\langle 80 \mathrm{MPa}$, Meet the strength requirements.

For unit 36: $N=21.56 N$ 
$\sigma_{t}=\frac{N}{A}=\frac{21.56}{0.41}=52.9 \mathrm{MPa}\langle 70 \mathrm{MPa}$, Meet the strength requirements.

\section{Comparative Analyses}

\section{Comparison between Test and Structural Solver Results}

(1)The maximum displacement point of model is obtained in a cross position of longitudinal beam by the load test, consistent with the structural solver results.

(2)Displacement which is obtained by testing longitudinal span midpoint has certain deviation with displacement calculated by structural solver. It is mainly due to the presence of certain error when the structural solver simplifies the $3 \mathrm{D}$ spatial structure to plane structure. At the same time, the test operation and reading will cause error. But overall, the error is small and reasonable. It is in the range of experimental permitted.

(3)Under the same loads, the displacements of beam span's midpoints measured by test are slightly larger than structural solver's calculation. The ultimate bearing capacity obtained by model experiment is less than that of the structural solver. It is mainly due to unavoidable defects in production of materials and model making process.

\section{Comparative Analysis of Two Kinds of Model Structure}

(1)The truss aqueduct is single and convenient; The cable-stayed aqueduct has complicated structure, large engineering quantity and long construction period, but it's beautiful enough.

(2)Under the same loads, deformation of cable-stayed aqueduct is smaller than that of truss aqueduct.

(3)When the two model reach failure, ultimate bearing capacity of cable-stayed aqueduct is greater than that of the truss aqueduct.

(4)The charge mass ratio: Truss aqueduct $\frac{F}{M}=\frac{48 \times 9.8}{443.5}=1.06$, cable-stayed aqueduct $\frac{F}{M}=\frac{57 \times 9.8}{482.2}=1.16$

\section{Conclusion}

By the reasonable adjustment of cable-stayed type through the cable stress, the longitudinal force and deformation conditions can be effectively improved to achieve reasonable design and reduce material consumption. Cable-stayed aqueduct's charge mass ratio is greater than that of the truss aqueduct, it saves material as well. So for the long span aqueduct, it is better to use cable-stayed aqueduct. It not only can solve the problems of huge load, and has the advantages of beautiful appearance and ornamental. It can make the safety, economy, beautiful appearance and practicability of harmony and unity.

\section{References}

[1]C.G.Wu. Hydraulics [M]. Beijing, Higher Education Press, 2008:190-209.

[2]H.Mei, C.K.Jia, X.X.Zhou.Cable-stayed aqueduct design [J]. Northwest Hydroelectric Power. 2006(S1).

[3]H.L.Yan. Hydraulic structures [M]. Beijing, Chemical Industry Press, 2007:290-292.

[4]L.Chen, F.Liu.Design of arch rectangular truss aqueduct and static load test of model [J]. The people of the Yangtze River. 2011.

[5]C.Li,X.X.Wang,B.Ji.Calculation and Analysis on the design of cable-stayed bridge [J]. Communications Science and Technology Heilongjiang. 2001(06). 